\title{
Symmetric Hadron Pairs at Large Transverse Momenta as a Test of Hard Scattering Models
}

\author{
R. Baier, J. Engels, and B. Petersson \\ Department of Theoretical Physics, University of Bielefeld, D-4800 Bielefeld, Federal Republic of Germany
}

Received 24 April 1959

\begin{abstract}
The inclusive cross section of hadron pairs produced back-to-back with large transverse momenta is examined in the parton model. It is shown quantitatively that this cross section is determined directly by the hard scattering subprocesses, without being influenced by the internal momentum of the constituents, even for transverse momenta of the order of $2-3 \mathrm{GeV} / c$. The predictions of the phenomenological quark-quark scattering model and of the quantum chromodynamics model for the back-to-back cross section are compared with recent Fermi-lab data. Predictions are made for the corresponding cross section at ISR-energies.
\end{abstract}

\section{Introduction}

Investigations of hadron production at large transverse momenta in hadron-hadron collisions are important, because in the parton model they lead to otherwise not available information about the interaction of constituents [1]. In this paper we investigate the inclusive production of hadron pairs with large transverse momenta $\left(p_{T} \gtrsim 2 \mathrm{GeV} / c\right)$ in opposite hemispheres, essentially back-to-back in the centre of mass system of the incoming hadrons. We show quantitatively that this special configuration probes the hard, i.e. large angle, scattering of the hadron constituents even for transverse momenta of the secondaries of the order of $p_{T} \simeq 2-4 \mathrm{GeV} / c$. This is in contrast to the single particle inclusive spectra, for which the model calculations for $p_{T} \lesssim 6$ $\mathrm{GeV} / \mathrm{c}$ strongly depend on the treatment of the internal transverse momenta of the constituents and their scattering at low momentum transfer, for which assumptions outside the model are required.

In Section II qualitative and quantitative arguments are given, why the two particle back-to-back cross section reflects the $p_{T}$-dependence of the hard scattering subprocesses undisturbed by the internal momenta of the constituents. We show that a simple formula, which is the "hard scattering" limit of the full expression for the symmetric two particle cross section, reproduces the latter within $20-30 \%$ for $p_{T} \geqq 2 \mathrm{GeV} / c$.

There exist recent data of the Columbia-Fermi Lab-Stony Brook Collaboration $[2,3]$ measuring the double-arm differential cross section for different hadron pairs in proton-beryllium collisions. As a consequence of the above result, these data allow a critical test of hard scattering models.

In Section III the phenomenological quark-quark scattering model $[4,5]$ is investigated, especially from the point of view of determining empirically the quark-quark cross section (at least at $90^{\circ}$ ). The consistency with single particle spectra and the quantum number dependence is also discussed.

In Section IV the quantumchromodynamics model $[6-10]$ is examined. Simple scale-breaking parametrizations of structure- and fragmentation functions are derived. It is shown that this model may be consistent with the existing back-to-back data for mesons. The sensitivity of this result to the ingredients of the model is discussed. It is for example shown, that the constraints on the gluon distribution function resulting from a QCD analysis of recent neutrino data $[11,12]$, play a crucial role. Finally predictions for the back-to-back differential cross section at ISR energies are given.

\section{Symmetric Pair-Cross Section and Intrinsic Transverse Momenta of Partons}

\section{Qualitative Arguments}

We first give a simple argument to show, that the effects of the intrinsic transverse momenta $\left(q_{T}\right)_{p / h}$ of the partons in hadrons are expected to be much less important for the symmetric pair-cross section than for the single particle spectra [13]. For the 
a

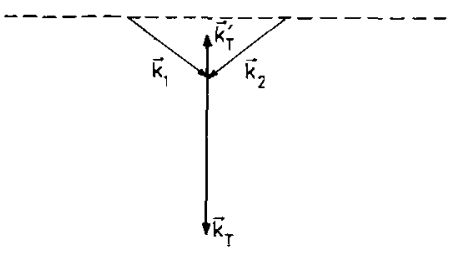

b

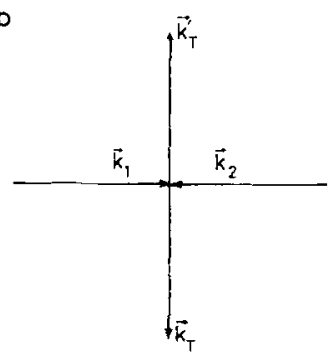

Fig. 1 a and $b$. Illustrations of constituent collisions relevant for the production of large $p_{T}$ hadrons. a collision for which the active constituents have internal transverse momenta, $\mathbf{b}$ collinear collision

phenomenological description of high $p_{T}$ processes a hard cross section of the type $d \sigma / d \hat{t}=1 / \hat{t}^{n} f\left(\hat{s}_{i} \hat{t}\right)$ and a distribution $F_{h}^{p}\left(\mathbf{q}_{T}\right)$-either of Gaussian or exponential form-damping large $\left(q_{T}\right)_{p / h}$ is applied. Triggering on a single large $p_{T}$-jet (parton) then two complementary mechanisms are present. The power behaved $d \sigma / d \hat{t}$ favours the production of the jet at low momentum transfer $\hat{t}$, but with large intrinsic $\left(q_{T}\right)_{p / h}$ for the active constituents, whereas the damping in $\left(q_{T}\right)_{p / h}$ prefers partons collinear with the initial hadrons. For the kinematical situation shown in Fig. 1a the invariants $\hat{s}$ and $\hat{t}$ are smaller than for the situation in Fig. 1b, when a jet with the same $p_{T}$ is produced. As a consequence the magnitude of the predicted single particle spectra depends crucially on the tail of the distribution in $\left(q_{T}\right)_{p / h}$, and on the recipes for cutting the singularities at $\hat{t}$ equal to zero. For intrinsic momenta $\left(q_{T}\right)_{p / h}$ of the order of one $\mathrm{GeV} / c$ the predictions for single particle spectra for $p_{T} \lesssim 6 \mathrm{GeV} / c$ depend on assumptions outside the hard scattering approach, as has been discussed by several authors $[5,6,14]$.

For the two-arm configuration one intuitively expects these biases to be minimal, since two opposite jets with large momenta have to be produced. The collinear configuration (Fig. 1b) is therefore favoured. One singular configuration with $\hat{t}=0$ can occur, namely when two partons happen to have equal and opposite large $\left(q_{T}\right)_{p / h}$ equal to the $p_{T}$ of the final state jets. The way of cutting out this singularity should not be important, since this extreme possibility is at least suppressed by the square of the distribution $F_{h}^{p}\left(\mathbf{q}_{T}\right)$. Therefore one expects the hard cross section to be relevant already for the production of a pair of hadrons with transverse momenta of about twice of $\left\langle q_{T}\right\rangle_{p / h}$ i.e. with $p_{T}$ of $2 \mathrm{GeV} / \mathrm{c}$. These expectations are in fact supported by the following quantitative test.

\section{Quantitative Analysis}

The cross section for the reaction $A+B \rightarrow C+D+X$ is given in the impulse approximation by

$$
\begin{aligned}
& E_{C} E_{D} \frac{d^{6} \sigma}{d^{3} p_{C} d^{3} p_{D}} \\
& =\int d z d z^{\prime} \int d^{2} q_{T} \int d^{2} q_{T}^{\prime} E_{k} E_{k^{\prime}} \frac{d^{6} \sigma^{A+B \rightarrow k+k^{\prime}}}{d^{3} k d^{3} k^{\prime}} \\
& \cdot \frac{\bar{z}}{z^{3}} D_{k}^{C}\left(z, \mathbf{q}_{T}\right) \frac{\bar{z}^{\prime}}{z^{\prime 3}} D_{k^{\prime}}^{D}\left(z^{\prime}, \mathbf{q}_{T}^{\prime}\right),
\end{aligned}
$$

where $D_{k}^{h}\left(z, \mathbf{q}_{T}\right)$ is the number of hadrons with momentum (energy) fraction $z=\mathbf{p} \cdot \mathbf{k} / \mathbf{k}^{2}(\bar{z}=$ $\left.\sqrt{z^{2}+\mathbf{q}_{T}^{2} / \mathbf{k}^{2}}\right)$ and transverse momentum $\mathbf{q}_{T}=\mathbf{p}-$ $z \mathbf{k}\left(\mathbf{q}_{T} \cdot \mathbf{k}=0\right)$ originating from a given constituent with momentum $\mathbf{k}$. The jet-jet cross section for producing two constituents with momenta $\mathbf{k}$ and $\mathbf{k}^{\prime}$, respectively, is calculated as

$$
\begin{aligned}
& E_{k} E_{k^{\prime}} \frac{d^{6} \sigma^{A+B \rightarrow k+k^{\prime}}}{d^{3} k d^{3} k^{\prime}} \\
& =\int \frac{d x_{1}}{\bar{x}_{1}} \frac{d x_{2}}{\bar{x}_{2}} \int d^{2} q_{1 T} d^{2} q_{2 T} F_{A}^{k_{1}}\left(x_{1}, \mathbf{q}_{1 T}\right) F_{B}^{k_{2}}\left(x_{2}, \mathbf{q}_{2 T}\right) \\
& \cdot \frac{\hat{s}}{2 \pi} \frac{d \sigma^{k_{1}+k_{2} \rightarrow k+k^{\prime}}}{\overline{d \hat{t}}}(\hat{s}, \hat{t}) \delta^{4}\left(k_{1}+k_{2}-k-k^{\prime}\right),
\end{aligned}
$$

with $F_{h}^{k}\left(x, \mathbf{q}_{T}\right)=\bar{x} G_{h}^{k}\left(x, \mathbf{q}_{T}\right)$ and $G_{h}^{k}\left(x, \mathbf{q}_{T}\right)$ is the number of constituents of type $k$ within a hadron $h$ with momentum $\mathbf{p}\left(\mathbf{k}=x \mathbf{p}+\mathbf{q}_{T}, \mathbf{p} \cdot \mathbf{q}_{T}=0\right.$ and $\bar{x}=$ $\sqrt{x^{2}+\mathbf{q}_{T}^{2} / \mathbf{p}^{2}}$ ). We further assume the partons to have zero mass and to be on mass shell.

For the following we make the factorization ansatz

$$
\begin{aligned}
& F_{h}^{k}\left(x, \mathbf{q}_{T}\right)=f_{h}^{k}(x) \frac{1}{\pi\left\langle q_{T}^{2}\right\rangle_{p / h}} \exp \left(-\frac{q_{T}^{2}}{\left\langle q_{T}^{2}\right\rangle_{p / h}}\right), \\
& D_{k}^{h}\left(z, \mathbf{q}_{T}\right)=D_{k}^{h}(z) \frac{1}{\pi\left\langle q_{T}^{2}\right\rangle_{h / p}}-\exp \left(-\frac{q_{T}^{2}}{\left\langle q_{T}^{2}\right\rangle_{h / p}}\right),
\end{aligned}
$$

and take as active partons the quarks only. One can now fix $\left\langle q_{T}^{2}\right\rangle_{h / q}=0.25 \mathrm{GeV}^{2} / c^{2}$, as it is measured via the jet analysis of electron-positron annihilation $[15,16]$. For the intrinsic quark transverse momentum we vary $\left\langle q_{T}^{2}\right\rangle_{q / h}$ from $0.25 \mathrm{GeV}^{2} / c^{2}$ up to $1 \mathrm{GeV}^{2} / c^{2}$, where this large upper limit comes from the interpretation of the dimuon high mass data in terms of the quark annihilation model [17]. The structure and the fragmentation functions, $f_{h}^{q}(x)$ and $D_{q}^{h}(z)$, 
respectively, are taken in scaling form from standard fits $[18,19]$ to lepton data. When the hadrons are produced at $90^{\circ}$-with rapidities $y_{C}=y_{D}=0$ - the hard scattering cross section $d \sigma / d \hat{t}$ is essentially probed at angles around $90^{\circ}$, i.e. $\hat{t}=\hat{u}=-\hat{s} / 2$. For that reason we use the following simple ansatz [4],

$\frac{d \sigma}{d \hat{t}}=\frac{\text { const. }}{\left(k_{T}^{2}+m_{0}^{2}\right)^{n}}, \quad k_{T}^{2}=\frac{\hat{t} \hat{u}}{\hat{s}}\left(\simeq \frac{|\hat{t}|}{2}\right.$ around $\left.90^{\circ}\right)$.

In order to get a strong effect due to the trigger bias discussed above a high power $n=4.5$ is taken. The eight-dimensional integral $(1,2)$ is evaluated by a Monte Carlo program.

The dependence of the symmetric pair-cross section $E_{C} E_{D} \frac{d^{6} \sigma}{d^{3} p_{C} d^{3} p_{D}}$ at $90^{\circ}$, i.e. with $\left|\mathbf{p}_{T C}\right|=\left|\mathbf{p}_{T D}\right|=p_{T}$, $y_{C}=y_{D}=0, \varphi_{C}=0$ and $\varphi_{D}=\pi$, on the parameters $m_{0}^{2}$ and $\left\langle q_{T}^{2}\right\rangle_{q / h}$, respectively, is shown in Fig. 2 for two energies $\sqrt{s}=19 \mathrm{GeV}$ and $27 \mathrm{GeV}$.

The first essential observation is that indeed the shape of the cross section as a function of $p_{T}$, $2 \leqq p_{T} \leqq 4 \mathrm{GeV} / \mathrm{c}$, is not changed when increasing

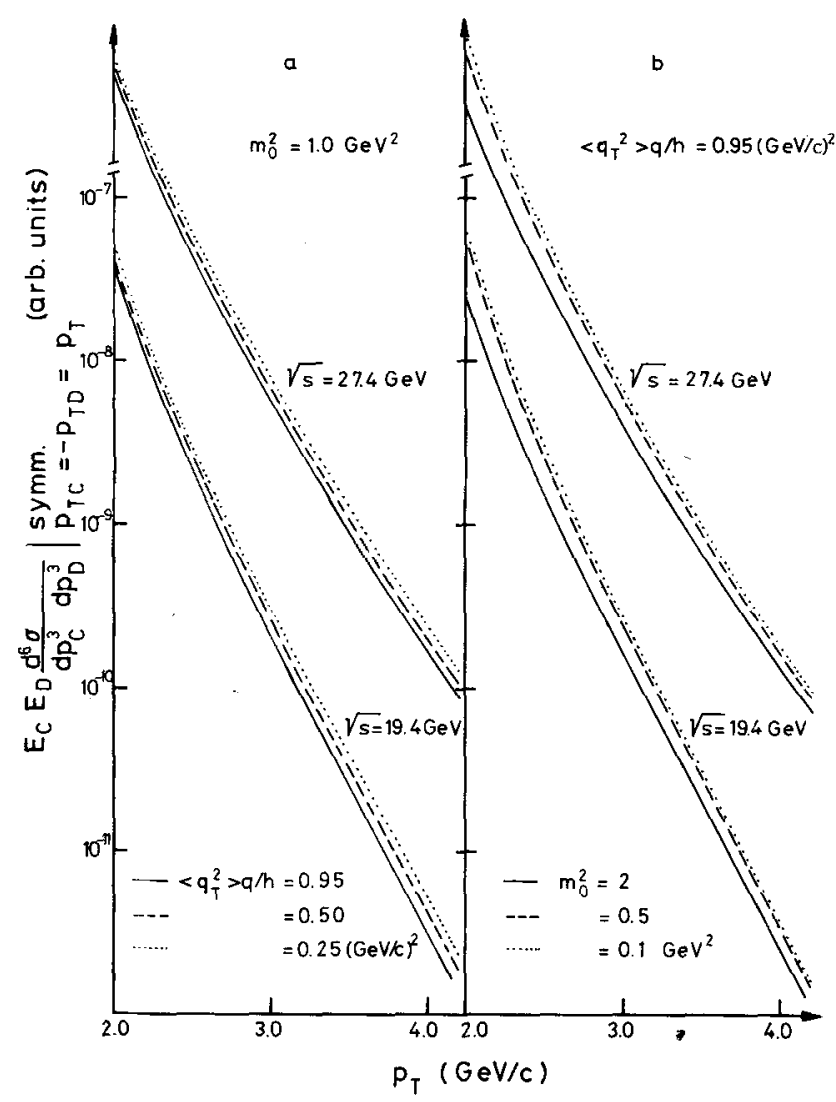

Fig. 2 and $b$. The dependence of the symmetric pair cross-section $E_{C} E_{D} \frac{d^{5} \sigma}{d^{3} p_{C} d^{3} p_{D}}$ as a function of $p_{T}=p_{T C}=-p_{T D}$ on the parameters $\left\langle q_{T}^{2}\right\rangle_{q / h}$ a and $m_{0}^{2}$ b for two different energies $\sqrt{ } s=19.4$ and $27.4 \mathrm{GeV}$ $\left\langle q_{T}^{2}\right\rangle_{g / h}$ from 0.25 up to $0.95 \mathrm{GeV}^{2} / c^{2}$, although the magnitude of $E_{C} E_{D} \frac{d^{6} \sigma}{d^{3} p_{C} d^{3} p_{D}}$ is decreasing (Fig. 2a). The ratio of the cross sections calculated with $\left\langle q_{T}^{2}\right\rangle_{q / h}=0.25 \mathrm{GeV}^{2} / c^{2}$ and $0.95 \mathrm{GeV}^{2} / c^{2}$ are, however, constant within $20 \%$ between $p_{T}=2 \mathrm{GeV} / c$ and $p_{T}=4 \mathrm{GeV} / c$. The variation with respect to $m_{0}^{2}$ has a slightly bigger effect (Fig. 2b): changing $m_{0}^{2}$ from $2 \mathrm{GeV}^{2}$ to $0.1 \mathrm{GeV}^{2}$ the cross section increases by a factor of three at $p_{T}>\simeq 2 \mathrm{GeV} / c$, but for $p_{r}>2.5 \mathrm{GeV} / c$ again the shape of the pair-cross section becomes fairly independent of the cut-off parameter $m_{0}^{2}$.

We will now show that a simple expression for $E_{C} E_{D} \frac{d^{6} \sigma}{d^{3} p_{C} d^{3} p_{D}}$ derived in the "hard scattering" limit, namely $\left\langle q_{T}\right\rangle_{q / h} \rightarrow 0$ and $\left\langle q_{T}\right\rangle_{h / q} \rightarrow 0$, gives a good approximation to the full expression (1) and (2). In this limit one has

$$
\begin{aligned}
& \left.E_{C} E_{D} \frac{d^{6} \sigma}{d^{3} p_{C} d^{3} p_{D}}\right|_{\substack{y_{C}=y_{D}=0,\left|p_{T C}\right|>\left|p_{T D}\right| \\
\varphi_{C}=0, \varphi_{D}=\pi\left(p_{\text {out }}=0\right)}} \\
& =\frac{1}{\pi} \frac{1}{p_{T C}} \int_{x_{T}}^{1} \frac{d z}{z} f_{A}^{k_{1}}\left(x=\frac{x_{T}}{z}\right) f_{B}^{k_{2}}(x) \frac{d \sigma^{k_{1}+k_{2} \rightarrow k+k^{\prime}}}{d \hat{t}} \\
& \left(\hat{s}=4 p_{T C}^{2} / z^{2}, \hat{t}=\hat{u}\right) D_{k}^{C}(z) D_{k^{\prime}}^{D}\left(x_{E} z\right) g\left(p_{\text {out }}=0\right),
\end{aligned}
$$

where $x_{T}=\frac{2 p_{T C}}{\sqrt{ } s}$ and $x_{E}=\frac{\left|p_{T D}\right|}{\left|p_{T C}\right|}$. The momentum $p_{\text {out }}$ is the momentum of particle $D$ perpendicular to the plane defined by the incoming particles and particle $C$. In case of neglecting the intrinsic momenta $\left(\mathbf{q}_{T}\right)_{q / h}$ and $\left(\mathbf{q}_{T}\right)_{h / q}$ the $p_{\text {out }}$-distribution is given by $g\left(p_{\text {out }}\right)=\delta\left(p_{\text {out }}\right)$. With Gaussian distributions for $\left(q_{T}\right)_{q / h}$ and $\left(q_{T}\right)_{h / q}$, however, one finds

$$
\begin{aligned}
& g\left(p_{\text {out }}\right)=\frac{1}{\sqrt{2 \pi\left\langle p_{\text {out }}^{2}\right\rangle}} \exp \left(-\frac{p_{\text {out }}^{2}}{2\left\langle p_{\text {out }}^{2}\right\rangle}\right), \\
& \int_{-\infty}^{+\infty} g\left(p_{\text {out }}\right) d p_{\text {out }}=1,
\end{aligned}
$$

where the mean square of $p_{\text {out }}$ is derived as

$$
\left\langle p_{\text {out }}^{2}\right\rangle=\frac{1}{2}\left[2\left\langle q_{T}^{2}\right\rangle_{q / h} x_{E}^{2} z^{2}+\left\langle q_{T}^{2}\right\rangle_{h / q}\left(1+x_{E}^{2}\right)\right] \text {. }
$$

This result follows either from simple geometrical considerations (see Fig. 3) or from calculating via saddle point methods the leading term of the full expression $(1,2,3)$ in the limit $\left\langle q_{T}^{2}\right\rangle_{q / h} \rightarrow 0$ and $\left\langle q_{T}^{2}\right\rangle_{h / q} \rightarrow 0$.

In expression (5) the dependence of the pair-cross section on $\left\langle q_{T}^{2}\right\rangle_{q / h}$ is now contained in $g\left(p_{\text {out }}=0\right)$.

The dependence of the cross section $E_{C} E_{D} \frac{d^{6} \sigma}{d^{3} p_{C} d^{3} p_{D}}$ on $p_{T}$ calculated from (5) for the symmetric configuration is shown in Fig. 4, and it is compared with the result calculated from the full expression $(1,2)$. 
a $\left(\vec{a}_{T}\right)_{h / q}=0$

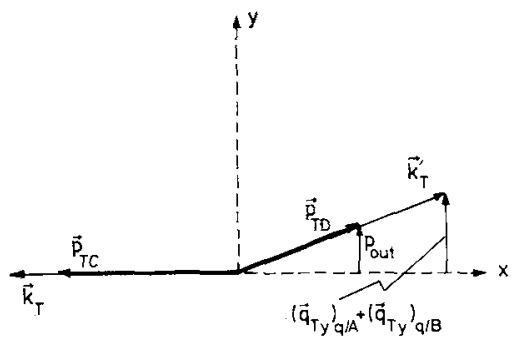

b $\left(\vec{q}_{T}\right)_{q / h}=0$

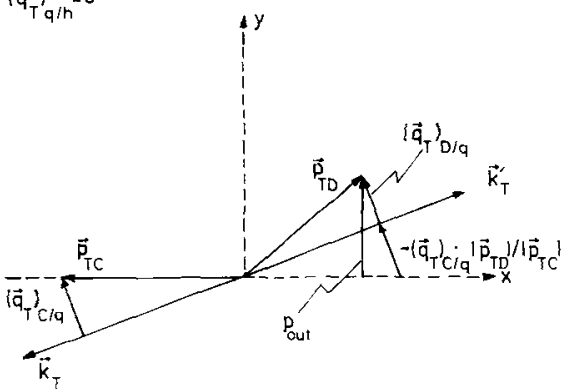

Fig. $3 \mathrm{a}$ and $\mathbf{b}$. Illustration of the different terms appearing in (7). $\mathbf{a}$ for the case $\left(\mathbf{q}_{T}\right)_{h / q}=0$ and $\mathbf{b}$ for $\left(\mathbf{q}_{t}\right)_{q / h}=0$

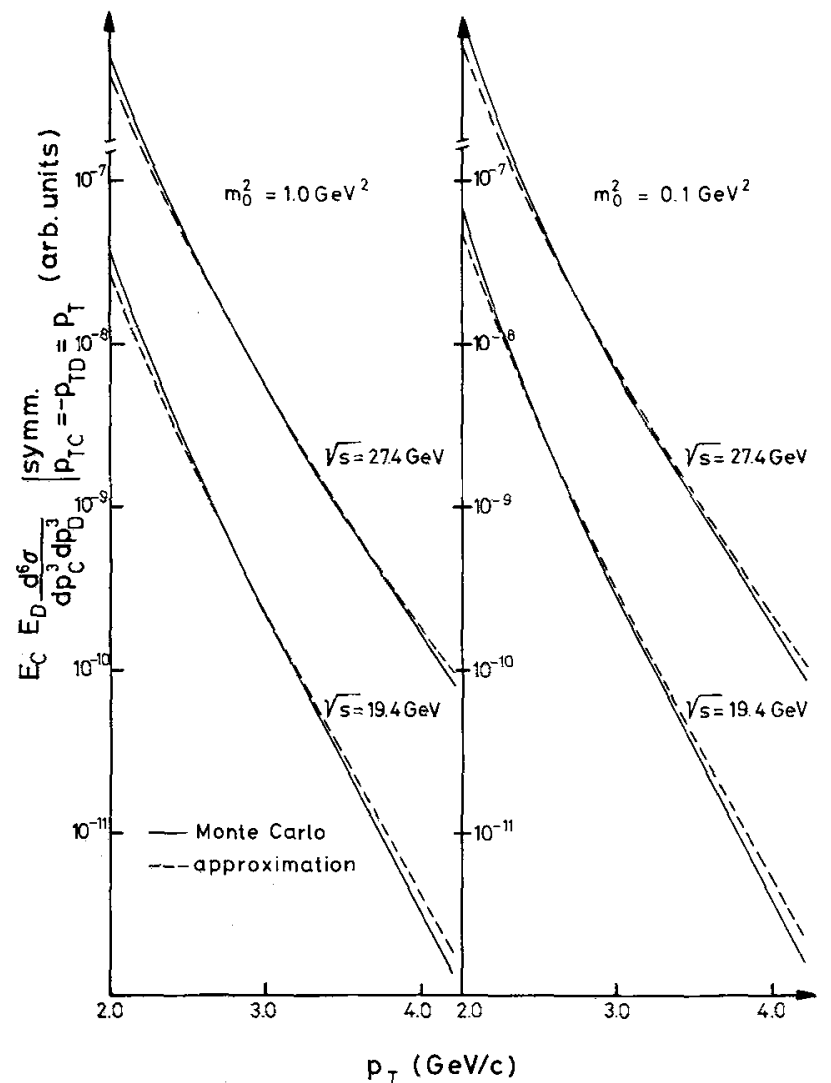

Fig. 4. Comparison of the $p_{T}$ dependence of the symmetric pair cross-section $E_{C} E_{D} \frac{d^{3} \sigma}{d^{3} p_{C} d^{3} p_{D}}$ calculated from the expression $(1,2)$-solid curve-and from the "hard scattering" approximation (5)-dashed curve $\left(\left\langle q_{T}^{2}\right\rangle_{q / h}=0.95 \mathrm{GeV}^{2} / c^{2},\left\langle q_{T}^{2}\right\rangle_{h / q}=0.25\right.$ $\left.\mathrm{GeV}^{2} / \mathrm{c}^{2}\right)$
It turns out that the "hard scattering" approximation is indeed an excellent one-the deviation from the Monte Carlo result is less than $15 \%-$. Thus for the symmetric configuration of opposite hadron pairs it is not necessary to perform time consuming multidimensional Monte Carlo integrations, only a onedimensional integral has to be done. Furthermore, as one can see from (5), in this configuration - even for $\left|p_{T C}\right| \neq\left|p_{T D}\right|-$ the cross section is sensitive to the behaviour of the constituent two-body interaction only at $\hat{t}=\hat{u}$, i.e. at $90^{\circ}$.

From this investigation we are confident that one can apply the "hard scattering" approximation for most high $p_{r}$ data on opposite side hadron pairs. It should be stressed again that this is not the case for single particle spectra, where predictions are uncertain within factors up to ten for $p_{T} \lesssim 5 \mathrm{GeV} / c$ $[5,6]$.

We will now use the formula (5) to compare two models with the recent FNAL-Stony Brook-Columbia data on hadron pairs $[2,3]$.

\section{Phenomenological Quark-Quark Scattering Model}

In this model $[4,5]$ one assumes that quark-quark collisions dominate the production of high transverse momentum particles. The structure functions of quarks within the initial hadrons $f_{h}^{q}(x)$ and the fragmentation functions of quarks into hadrons $D_{q}^{h}(z)$ are determined (in scaling form) from experimental lepton data. For the following analysis the parametrizations for $f_{h}^{q}(x)$ and $D_{q}^{h}(z)$ are used as given in [18] and [19], respectively. The elastic quark-quark scattering cross-section $d \sigma / d \hat{t}=1 / \hat{t}^{n} f(\hat{s} / \hat{t})$ should be determined empirically from some set of large $p_{T}$ data, and the model should then give a consistent description of all large $p_{T}$ data.

As discussed above the effects due to the internal motion of quarks within hadrons are absent in the pair-cross section. Therefore the dependence of $E_{C} E_{D} \frac{d^{6} \sigma}{d^{3} p_{C} d^{3} p_{D}}$ as a function of $p_{T}=\left|\mathbf{p}_{T C}\right|=\left|\mathbf{p}_{T D}\right|$ with fixed $x_{T}=2 p_{T} / \sqrt{ } s$-allows one to deduce directly the power $n$ and the normalization. Since the data on the pair-cross section only exist at $90^{\circ}$, we parametrize the quark-quark scattering in the form (see (4))

$$
\frac{d \sigma}{d \hat{t}}=\frac{B}{\left(k_{T}^{2}+1\right)^{n}} .
$$

For constant $\left.\left\langle q_{T}^{2}\right\rangle_{q / h}\right\rangle\left\langle q_{T}^{2}\right\rangle_{h / q}$ this corresponds according to (5) to

$\left.E_{C} E_{D} \frac{d^{6} \sigma}{d^{3} p_{C} d^{3} p_{D}}\right|_{\text {symm. }}=\frac{1}{p_{T}^{n_{\text {pair }}}} \frac{\tilde{f}\left(x_{T}\right)}{\sqrt{\left\langle q_{T}^{2}\right\rangle_{q / h}}}$,

with $n_{\text {pair }}=2 n+1$. The single particle cross section 


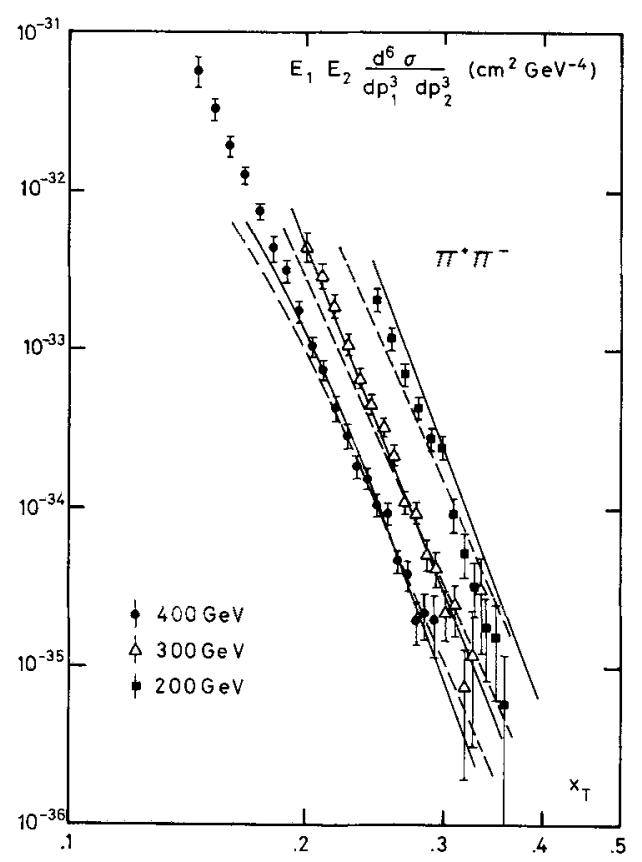

Fig. 5. Comparison of the data [2] for the symmetric pair crosssection versus $x_{T}$ of the process $p+B e \rightarrow \pi^{+} \pi^{-}+X$ at three different energies $\left(p_{\mathrm{lab}}=200,300\right.$ and $\left.400 \mathrm{GeV} / \mathrm{c}\right)$ with the phenomenological quark-quark scattering model. Two hard crosssections $d \sigma / d \hat{t}$ are chosen, $d \sigma / d \hat{t}=\frac{60 \mathrm{mb} \mathrm{GeV}^{7}}{\left(k_{\mathrm{T}}^{2}+1\right)^{4.5}}$ (solid curve) and $d \sigma / d \hat{t}=\frac{3.4 \mathrm{mb} \mathrm{GeV}^{5}}{\left(k_{T}^{2}+1\right)^{3.5}}$ (dashed curve). The curves are evaluated
at $y=0$

behaves as

$E \frac{d^{3} \sigma}{d^{3} p}=\frac{\tilde{g}\left(x_{T}\right)}{p_{T}^{n_{\text {single }}}}$

with $n_{\text {single }}=2 n$, when the internal transverse momenta of quarks are neglected.

In Fig. 5 the model is compared with the data [2] on $\left.E_{C} E_{D} \frac{d^{6} \sigma}{d^{3} p_{C} d^{3} p_{D}}\right|_{\text {symm. }} \quad$ for the process $p+B e \rightarrow \pi^{+}+$ $\pi^{-}+X$ at three different energies $\left(p_{1 \mathrm{ab}}=200,300\right.$ and $400 \mathrm{GeV} / c$ ). The solid curves correspond to $n=4.5$ and $B=60 \mathrm{mb} \mathrm{GeV}^{7}$, the dashed ones to $n=3.5$ and $B=3.4 \mathrm{mb} \mathrm{GeV}^{5}$. When the power $n$ is fixed, the $x_{T}$ dependence is predicted by the model. The parameter $B$ is fixed by the data point with $x_{T}=0.24$ at $p_{1 a b}=400 \mathrm{GeV} / c$. The values used for the mean squares of the transverse momenta are $\left\langle q_{T}^{2}\right\rangle_{q / h}=$ $0.95 \mathrm{GeV}^{2} / c^{2}$ and $\left\langle q_{T}^{2}\right\rangle_{h / q}=0.25 \mathrm{GeV}^{2} / c^{2}$.

For the comparison with the present data $[2,3]$ (Fig. 5) one should keep in mind that the measured cross sections are given for a beryllium nucleus, and that the data are averaged over bands in rapidity centered at $y=0.0,0.2$ and 0.4 for $p_{\text {lab }}=400,300$ and $200 \mathrm{GeV} / c$, respectively. The atomic number dependence is only measured at $p_{\text {lab }}=400 \mathrm{GeV} / c$, where it is observed to be proportional to the atomic number $A$ for the symmetric configuration of the $\pi^{+} \pi^{-}$pairs [20]. In the model calculations we assume the naive $A$ dependence proportional to $A^{1}$ at all energies. The curves in Fig. 5 are all evaluated at $y=0$. The effect of changing the rapidity from $y=0$ to $y=0.2$ at $p_{\text {lab }}=300 \mathrm{GeV} / c$ is insignificant; at $p_{\text {lab }}=200 \mathrm{GeV} / c$, however, the theoretical prediction at $y=0.4$ is smaller than the one at $y=0.0$ by a factor of 1.5 for the smaller $x_{T}$ region and by a factor 3 at $x_{T} \simeq 0.35$. One should note that this variation with respect to $y$ only depends on the structure functions, since the invariants $\hat{s}, \hat{t}$ and $\hat{u}$ stay unchanged when the rapidities of both particles are shifted by the same amount.

These uncertainties imply that very detailed conclusions can not be drawn. Nevertheless, a high power $n$ in the hard cross section seems to be needed, i.e. $n=4-4.5$. With the quark-quark cross section determined from these pair data, one could calculate the single particle spectra. However, in the "hard scattering" limit the power $n=4-4.5$ would be in agreement with data ( $n_{\text {single }} \simeq 8-9$, according to (9)), while the normalization would be a factor 3-4 to low $[4,5]$. A naive inclusion of internal transverse momenta would not help, because the spectra would become steeper, although the effective normalization would increase. Of course, changing the power $n$ from 4.5 to 3.5 the ratio of the pair-cross sections (8) between $p_{\text {lab }}=200$ and $400 \mathrm{GeV} / c$ changes only by a factor of 2 at fixed $x_{T}$, and then there would be place for "smearing" effects to improve the single particle spectra. In order to quantify the above remarks, more data on pair-cross sections at widely separated energies are needed.

Since in the quark-quark model there is assumed to be no (flavour) quantum number exchange between the active quarks the quantum number correlations for different hadron pairs can be uniquely predicted once the functions $f_{h}^{q}$ and $D_{q}^{h}$ are determined. However, the determination of the fragmentation functions for strange particles from $e^{+} e^{-}$-data and deep inelastic data are not consistent, as discussed by Sehgal [19]. We use his parametrization for $D_{q}^{K}(z)$, but with the normalization increased for a factor of two, in order to get better agreement with the $e^{+} e^{-} \rightarrow K^{ \pm} X$ data [21] for $z \geqq 0.5$, which is the region relevant for our analysis.

In Fig. 6 the results for $\pi^{+} \pi^{-}, K^{+} \pi^{-}$and $\pi^{+} K^{-}$ pairs are compared with the data of [3] at $p_{\text {lab }}=$ $400 \mathrm{GeV} / c$ as a function of $m^{\prime}=\left|\mathbf{p}_{T C}\right|+\left|\mathbf{p}_{T D}\right|$ and $\Delta p_{T}=\left|\mathbf{p}_{T C}\right|-\left|\mathbf{p}_{T D}\right|$ (particle $C$ is the positively charged one). The parameters used are $n=4.5$ and $B=60 \mathrm{mb} \mathrm{GeV}^{7}$. The difference in the shapes for the different pairs as a function of $\Delta p_{T}$ is determined by the fragmentation functions. The agreement is in general good. As the model has approximate factorization we predict $\sigma\left(K^{+} K^{-}\right) \simeq \frac{\sigma\left(\pi^{+} K^{-}\right)}{\sigma\left(\pi^{+} \pi^{-}\right)} \sigma\left(K^{+} \pi^{-}\right)$, which is also in good agreement with the atomic number corrected data presented in Fig. 25 of [3]. 

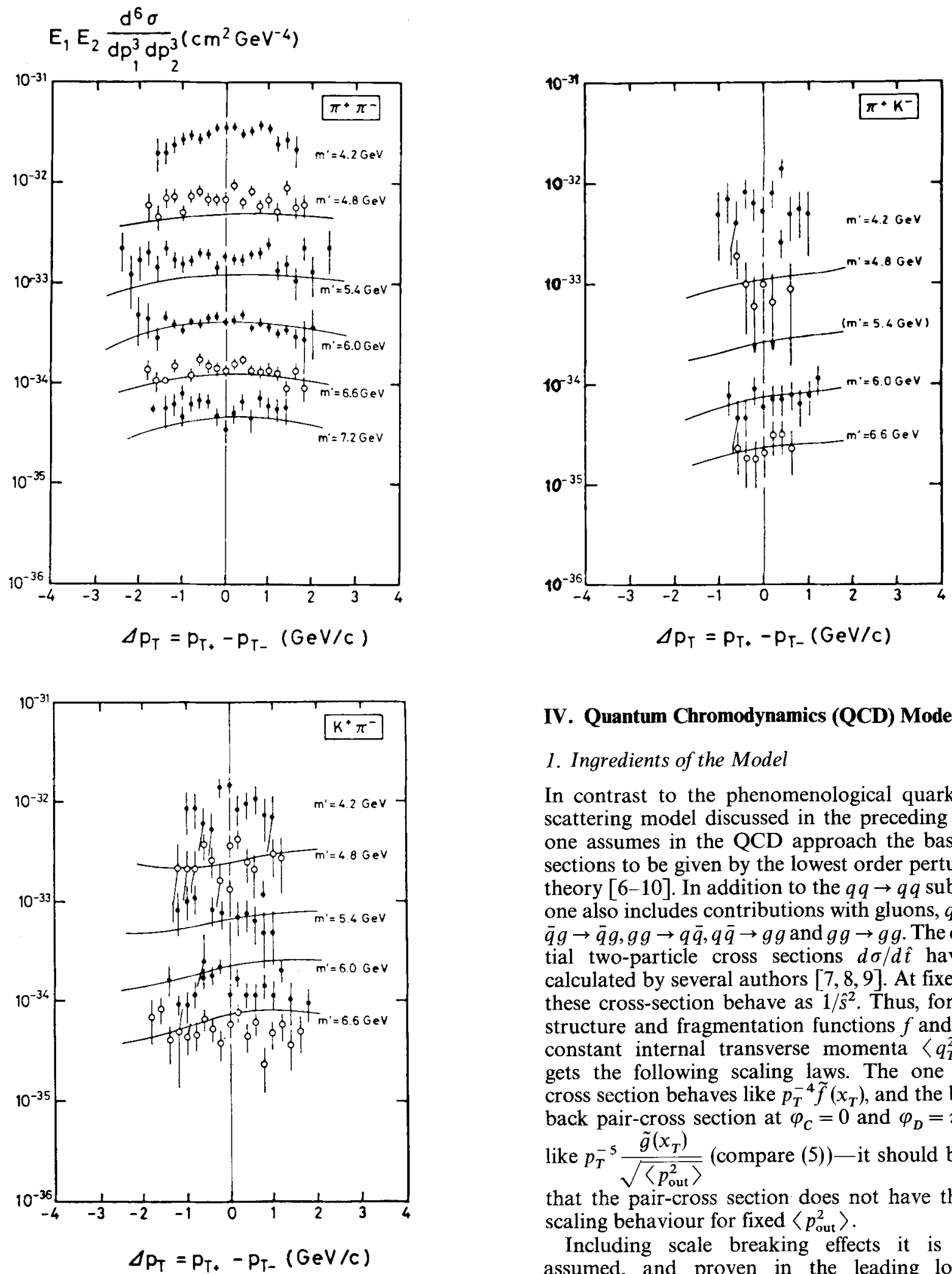

Fig. 6. Comparison of the data [3] for the pair cross-sections for $\pi^{+} \pi^{-}, K^{+} \pi^{-}$and $\pi^{+} K^{-}$pairs as a function of $m^{\prime}=p_{T C}+p_{T D}$ and $\Delta p_{T}=p_{T C}-p_{T D}$ in proton-beryllium collisions at $p_{\mathrm{lab}}=$ $400 \mathrm{GeV} / c$ with the phenomenological quark-quark scattering model, $d \sigma / d \hat{t}=60 \mathrm{mb} \mathrm{GeV}^{7} /\left(k_{T}^{2}+1\right)^{4.5}$

\section{Quantum Chromodynamics (QCD) Model}

\section{Ingredients of the Model}

In contrast to the phenomenological quark-quark scattering model discussed in the preceding section, one assumes in the QCD approach the basic cross sections to be given by the lowest order perturbation theory [6-10]. In addition to the $q q \rightarrow q q$ subprocess one also includes contributions with gluons, $q g \rightarrow q g$, $\bar{q} g \rightarrow \bar{q} g, g g \rightarrow q \bar{q}, q \bar{q} \rightarrow g g$ and $g g \rightarrow g g$. The differential two-particle cross sections $d \sigma / d \hat{t}$ have been calculated by several authors $[7,8,9]$. At fixed angle, these cross-section behave as $1 / \hat{s}^{2}$. Thus, for scaling structure and fragmentation functions $f$ and $D$ (and constant internal transverse momenta $\left\langle q_{T}^{2}\right\rangle$ ) one gets the following scaling laws. The one particle cross section behaves like $p_{T}^{-4} \tilde{f}\left(x_{T}\right)$, and the back-toback pair-cross section at $\varphi_{C}=0$ and $\varphi_{D}=\pi$, scales like $p_{T}^{-5} \frac{\tilde{g}\left(x_{T}\right)}{\sqrt{\left\langle p_{\text {out }}^{2}\right\rangle}}$ (compare (5))-it should be noted that the pair-cross section does not have the naive scaling behaviour for fixed $\left\langle p_{\text {out }}^{2}\right\rangle$.

Including scale breaking effects it is usually assumed, and proven in the leading logarithm approximation [22], that the inclusive quantities may be factorized into the cross section $d \sigma / d \hat{t}$ given by the Born term but with a running coupling constant, and scale breaking structure and fragmentation 
functions, which are universal, i.e. they can be taken from other experiments. We thus assume that the formula (5) for the pair-cross section can be written as

$$
\begin{aligned}
& \left.E_{C} E_{D} \frac{d^{6} \sigma}{d^{3} p_{C} d^{3} p_{D}}\right|_{y C=y D=0, p_{T C}>\left|p_{T D}\right|, p_{\text {out }}=0} \\
& =\frac{1}{\pi} \frac{1}{p_{T C x_{T}}} \int_{x_{T}}^{1} \frac{d z}{z} g_{1}\left(p_{\text {out }}=0\right) \sum_{\substack{\text { quarks, } \\
\text { gluon }}} f_{A}^{k_{1}}\left(x, Q^{2}\right) f_{B}^{K_{2}}\left(x, Q^{2}\right) \\
& \frac{d \sigma^{k_{1}+k_{2} \rightarrow k+k^{\prime}}}{d \hat{t}}\left(\hat{s}, \alpha_{s}\left(Q^{2}\right)\right) D_{k}^{C}\left(z, Q^{2}\right) D_{k^{\prime}}^{D}\left(x_{E} z, Q^{2}\right) .
\end{aligned}
$$

In the last equation we assume that quarks and gluons have the same intrinsic mean square transverse momenta i.e. $\left\langle q_{T}^{2}\right\rangle_{q / h}=\left\langle q_{T}^{2}\right\rangle_{g / h}$ and $\left\langle q_{T}^{2}\right\rangle_{h / q}=$ $\left\langle q_{T}^{2}\right\rangle_{h / g}$. The relation between the variable $Q^{2}$, as measured in other processes, and the invariants of the hard scattering $\hat{s}$ and $\hat{t}$ is still undetermined.

Several authors have analysed the single particle spectra in terms of the QCD model [6-10], and they have shown that scale breaking effects in $\alpha_{s}$, $f$ and $D$ may change the naive $p_{T}^{-4}$ scaling to an effective $p_{T}^{-6}$ behaviour (for not too large $p_{T}$ ). However, if the quarks and gluons have large primordial momenta of the order of $1 \mathrm{GeV} / c$ one expects a further steepening of the slope $E d \sigma / d^{3} p$ for medium $p_{T}\left(p_{T} \lesssim 5 \mathrm{GeV} / c\right)$, at least if one uses on-shell kinematics. Thus one can get agreement with the observed $p_{T}^{-8}$ behaviour and with the magnitude of the single-particle spectra. The latter calculation is nevertheless quite sensitive to the unknown cut-off parameters and to the shape of the "primordial" transverse momentum distribution.

As we have shown in Section II that the back-toback pair cross-section is insensitive to these effects it is extremely interesting to explore the QCD approach for these cross sections.

In expression (10) we use as input structure and fragmentation functions determined in the following way. For the structure functions $F_{2}\left(x, Q^{2}\right)$ and $x F_{3}\left(x, Q^{2}\right)$ it has been shown from the analysis of the recent measurements in high energy neutrino interactions that the $Q^{2}$-dependence is consistent with QCD $[11,12]$. We use these data and their parametrization as given by the CERN-DortmundHeidelberg-Saclay Collaboration in [12]. The valence distribution $x F_{3}$ is parametrized in analogy with the proposal of Buras and Gaemers [23] as follows

$$
\begin{aligned}
& x F_{3}\left(x, Q^{2}\right)=(x q(x)-x \bar{q}(x)) \\
& =\frac{3}{B\left(\eta_{1}^{V}, \eta_{2}^{V}+1\right)} x^{\eta_{1}^{V}(s)}(1-x)^{\eta_{2}^{V}(\bar{s})},
\end{aligned}
$$

with

$\eta_{1}^{V}=0.56-0.92 \cdot \frac{4}{25} \bar{s}$

$\eta_{2}^{V}=2.71+5.08 \cdot \frac{4}{25} \bar{s}$, where

$\bar{s}=\ln \left(\frac{\ln Q^{2} / \Lambda^{2}}{\ln Q_{0}^{2} / \Lambda^{2}}\right)$.

In the analysis of [12] it is concluded that with $Q_{0}^{2}=5 \mathrm{GeV}^{2} / c^{2}$ values of $A$ in the range $A=0.47 \pm$ $0.11 \mathrm{GeV}( \pm 0.1$ systematic error $)$ are allowed. Furthermore from the measurements of $F_{2}\left(x, Q^{2}\right)$ the total sea contribution is deduced

$x \bar{q}\left(x, Q^{2}\right)=A(\bar{s})(1-x)^{P(\bar{s})}$,

with the initial values at $\bar{s}=0$

$A(0)=0.99 \pm 0.07$,

$P(0)=8.1 \pm 0.7$.

From this and the QCD moment equations [24] the authors of [12] have also determined $A(\bar{s}), P(\bar{s})$ and the second and third gluon moments. For our analysis, however, we need an explicit parametrization of the gluon distribution function. We make therefore the ansatz

$x G\left(x, Q^{2}\right)=c_{g}(\bar{s}) x^{\eta_{1}^{g}(\bar{s})}(1-x)^{\eta_{2}^{g}(s)}$,

and use as input at $Q_{0}^{2}=5 \mathrm{GeV}^{2} / c^{2}$

$c_{g}(0)=2.01, \eta_{1}^{g}(0)=0, \eta_{2}^{g}(0)=2.9$

One should note the low value of $\eta_{2}^{g}(0)$ compared to the naive guesses of $\eta_{2}^{g} \simeq 4-5$. This low value comes from our parametrization and the large value of the third gluon moment $M_{3}^{g}\left(Q_{0}^{2}=5\right)=0.105$ given in [12] $\left(\eta_{2}^{g}(0)=\frac{M_{2}^{g}(5)}{M_{3}^{g}(5)}-2\right)$.

From the QCD moment equations for the second and third moments, we get by a quadratic fit the following $\bar{s}$-dependence of the parameters

$$
\begin{aligned}
& A(\bar{s})=0.99+0.72 \bar{s}+0.96 \bar{s}^{2}, \\
& P(\bar{s})=8.10-1.49 \bar{s}+5.10 \bar{s}^{2}, \\
& c_{g}(\bar{s})=2.01-3.56 \bar{s}+1.98 \bar{s}^{2}, \\
& \eta_{1}^{g}(\bar{s})=-1.13 \bar{s}+0.48 \bar{s}^{2},
\end{aligned}
$$

where as input $\eta_{2}^{g}(s)=2.9+5.08 \frac{4}{25} \cdot \bar{s}$ is used. For $\bar{s}<1$ we checked that our ansatz is also in reasonable agreement with the moment equations with $4 \leqq n \leqq 8$.

The gluon distribution function obtained is plotted in Fig. 7 for different values of $Q^{2}(A=0.6 \mathrm{GeV})$. It should be stressed that the results for the large $p_{T}$ pair-cross section are quite sensitive to the variation of $G\left(x, Q^{2}\right)$ in the region $x \simeq 0.5-0.8$, and that our parametrization of $G\left(x, Q^{2}\right)(15)$ is certainly not unique. Since we need the distribution functions for the different flavours separately, we take $u_{V}\left(x, Q^{2}\right)=$ $2 d_{V}\left(x, Q^{2}\right)$ and $\bar{u}=\bar{d}=s=\bar{s}=\bar{q}\left(x, Q^{2}\right) / 6$.

For the fragmentation functions, whose $Q^{2}$-dependence should also satisfy QCD moment equations 


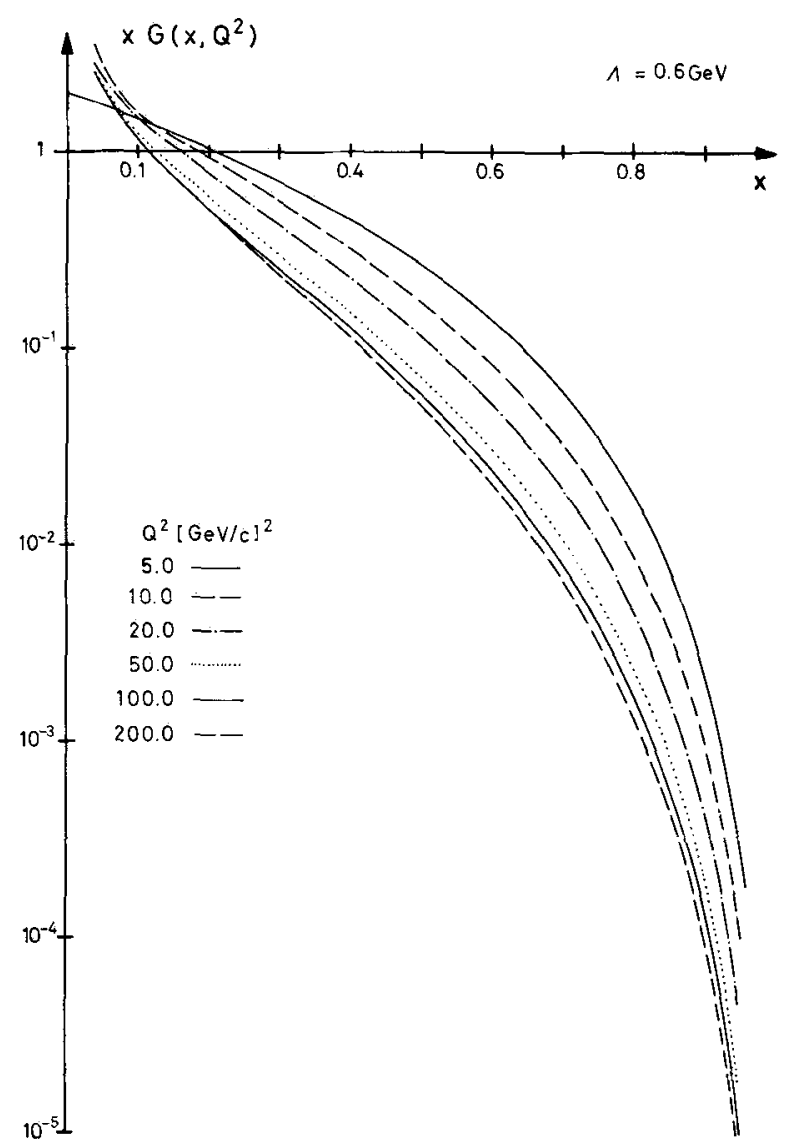

Fig. 7. The dependence on $x$ and $Q^{2}$ of the gluon structure function for $A=0.6 \mathrm{GeV}$ according to the parametrization described in the text

[25], the following parametrizations are assumed,

$D_{u}^{\pi^{-}}\left(z, Q^{2}\right)=\frac{1-z}{1+z} D_{u}^{\pi^{+}}\left(z, Q^{2}\right)=\frac{1-z}{2 z} D\left(z, Q^{2}\right)$,

$D\left(z, Q^{2}\right)=c_{q}(\bar{s}) z^{d^{q}(\bar{s})}(1-z)^{d_{2}^{q}(\bar{s})}$

$D_{g}^{\pi}\left(z, Q^{2}\right)=c_{g}(\bar{s}) z^{d_{1}^{g}(\bar{s})}(1-z)^{d_{2}^{g}(\bar{s})}$,

with input values chosen at $Q_{0}^{2}=25 \mathrm{GeV}^{2} / c^{2}$. This large value of $Q_{0}^{2}$ is chosen, because we believe that $\frac{1}{\sigma} \frac{d \sigma}{d z}\left(e^{+} e^{-} \rightarrow \pi X\right)$ starts to "scale" at $\sqrt{Q^{2}} \gtrsim 5 \mathrm{GeV} / c$. The input exponents are chosen according to conventional counting rules [26], the normalization of $D\left(z, Q_{0}^{2}\right)$ such that it is in agreement with the $e^{+} e^{-}$ data of $[15]$ for $z>0.4$. From the second and third QCD moment equations the following parameters in terms of a quadratic fit are obtained

$c_{q}(\bar{s})=0.50-0.07 \bar{s}+0.003 \bar{s}^{2}$,

$d_{1}^{q}(\bar{s})=-0.29 \bar{s}+0.06 \bar{s}^{2}$,

$d_{2}^{q}(\bar{s})=1.0+0.59 \bar{s}+0.05 \bar{s}^{2}$,

$c_{g}(\bar{s})=0.50-0.75 \bar{s}+0.38 \bar{s}^{2}$,

$d_{1}^{g}(\bar{s})=-1.0-0.97 \bar{s}+0.38 \bar{s}^{2}$,

$d_{2}^{g}(\vec{s})=2.0+0.59 \vec{s}+0.05 \bar{s}^{2}$.

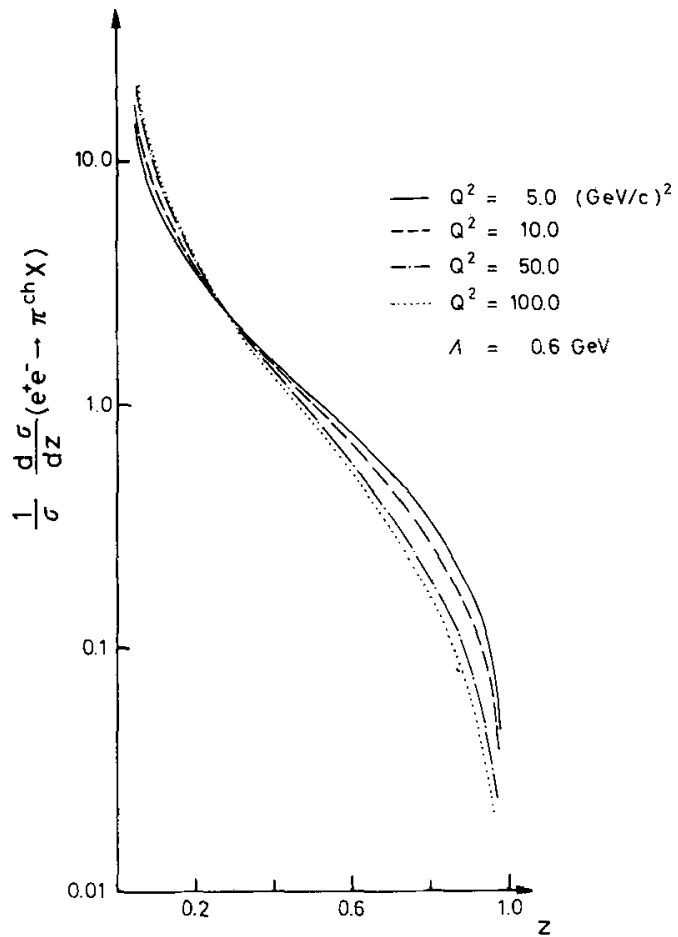

Fig. 8. The dependence on $z$ and $Q^{2}$ of the normalized crosssection $1 / \sigma d \sigma / d z$ for $e^{+} e^{-} \rightarrow$ charged pions $+X$ according to the parametrization described in the text

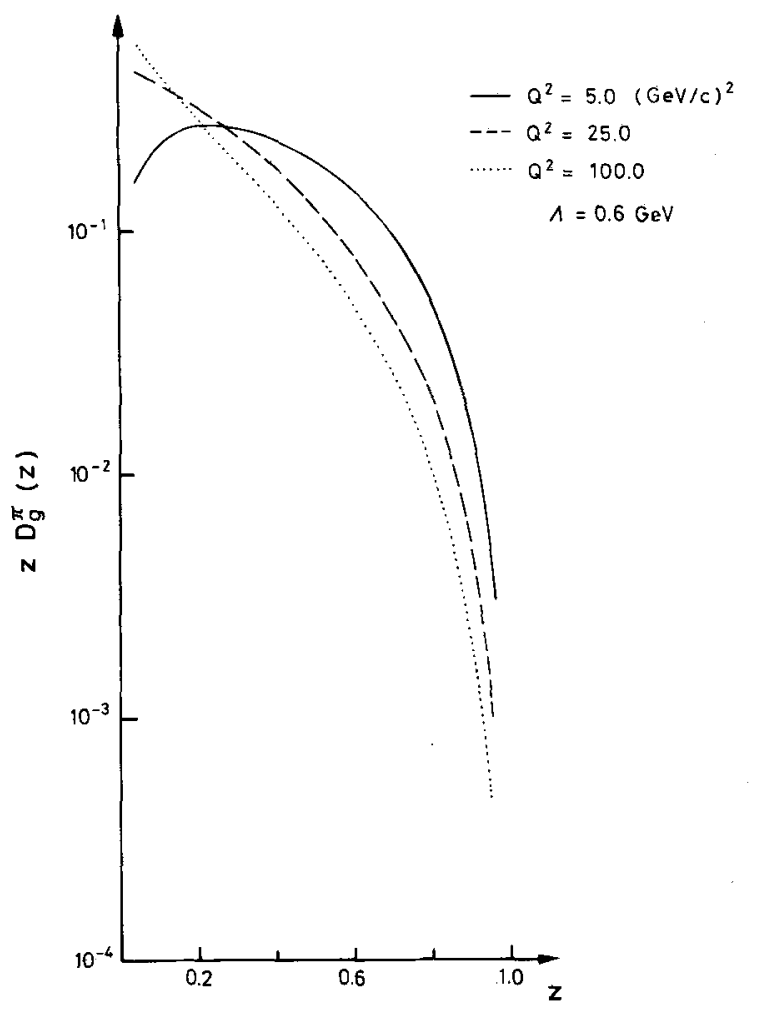

Fig. 9. The dependence on $z$ and $Q^{2}$ of the gluon fragmentation function $z D_{g}^{\pi}(z)$ according to the parametrization described in the text 
The cross section $\frac{1}{\sigma} \frac{d \sigma^{e^{+} e^{-\rightarrow \pi c h} X}}{d z}\left(z, Q^{2}\right)(=(2 / z-$ $\left.1 / 3) D\left(z, Q^{2}\right)\right)$ and the function $z D_{g}^{\pi}\left(z, Q^{2}\right)$ are plotted in Figs. 8 and 9, respectively. The (spurious) singularity which develops in (19) at $z=0$ is not directly important for the large $p_{T}$ application. What is significant, however, is the variation of the fragmentation functions with respect to $Q^{2}$ for $z \gtrsim 0.5$. One observes that the chosen parametrization gives a slow variation of $D\left(z, Q^{2}\right)$ in agreement with the absence of strong scaling violations in $e^{+} e^{-} \rightarrow \pi X$ $[15]$. The gluon fragmentation function, however, has a rather strong variation with $Q^{2}$.

In order to insert the above functions into (10) for the pair-cross section one has to choose an expression for $Q^{2}$ in terms of $\hat{s}$ and $\hat{t}$, and furthermore the parameter $A$ is free. For convenience the same relation as in [6] is used in the following,

$Q^{2}=\frac{2 \hat{s} \hat{t} \hat{u}}{\hat{s}^{2}+\hat{t}^{2}+\hat{u}^{2}}\left(=\frac{2}{3}|\hat{t}|\right.$ at $\left.90^{\circ}\right)$,

and for comparison the relation

$Q^{2}=\frac{\hat{t} \hat{u}}{\hat{s}}=k_{T}^{2}\left(=\frac{1}{2}|\hat{t}|\right.$ at $\left.90^{\circ}\right)$

is applied.

\section{Comparison with Symmetric Pair Data}

The comparison of the QCD-parametrization discussed above with the FNAL-data [2] for $\pi^{+} \pi^{-}$ pairs at the three different momenta $\left(p_{\text {lab }}=200\right.$, 300 and $400 \mathrm{GeV} / \mathrm{c}$ ) is presented in Fig. 10. We assume here the ratio of the nucleus to the nucleon cross section to be proportional to $A^{1}$. At $p_{\text {lab }}=200 \mathrm{GeV} / \mathrm{c}$ we plot the predictions at $y=0$ (solid curve) and at $y=0.4$ (dashed curve). At $p_{\text {lab }}=300 \mathrm{GeV} / c$ the difference between $y=0.0$ and $y=0.2$ is insignificant.

For the parameter $A$ we choose $A=0.6 \mathrm{GeV}$, which gives the best fit using relation (20). However, the normalization is sensitive to non-asymptotic effects; using relation (21) we have to change $A$ to $A=0.45 \mathrm{GeV}$ in order to fit the data equally well. These choices of $A$ correspond to a rather large coupling constant $\alpha_{s}$ in the lower range of $p_{T}$ of the data. For $p_{T} \simeq 2 \mathrm{GeV} / c$, where $|\hat{t}| \simeq$ $12-16 \mathrm{GeV}^{2}$, we get with $A=0.6 \mathrm{GeV}$ and (20) $\alpha_{s} \simeq 0.4\left(\alpha_{s}\left(Q^{2}\right)=\frac{12 \pi}{25 \ln Q^{2} / A^{2}}\right)$. The absolute normalization of the cross sections is approximately proportional to $\left(\left\langle q_{T}^{2}\right\rangle_{q, g / h}\right)^{-1 / 2}$; we fix $\left\langle q_{T}^{2}\right\rangle_{q, g ; h}=$ $0.95 \mathrm{GeV}^{2} / c^{2}$.

It can be seen from Fig. 10 that once the normalization is fixed, the $p_{\mathrm{T}}^{-5}$ behaviour of the Born terms is sufficiently modified by the scale breaking due to $\alpha_{s}\left(Q^{2}\right), f\left(x, Q^{2}\right)$ and $D\left(z, Q^{2}\right)$ to describe reasonably the shape of the steep $p_{T}$-spectra for $x_{T} \gtrsim 0.18$. One should note that in the scaling model one needs

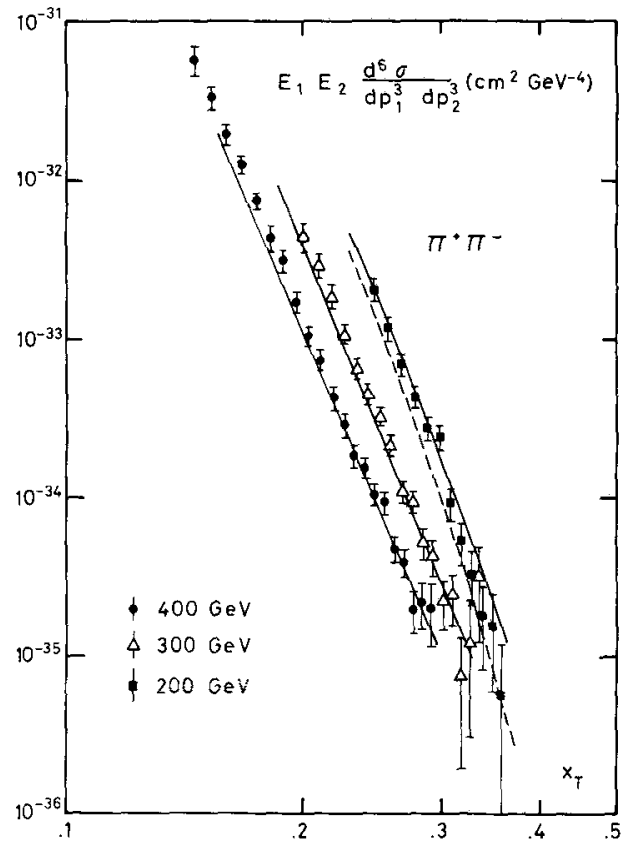

Fig. 10. Comparison of the data [2] for the symmetric pair-cross section versus $x_{r}$ of the process $p+B e \rightarrow \pi^{+}+\pi^{-}+X$ at three different energies $\left(p_{\mathrm{lab}}=200,300\right.$ and $400 \mathrm{GeV} / \mathrm{c}$ ) with the QCD model. The solid curves are calculated at $y=0$. The dashed curve is the prediction for $y=0.4$ at $p_{\text {lab }}=200 \mathrm{GeV} / c$

$n_{\text {pair }} \simeq 8-10$. Here one gets e.g. at $x_{T}=0.26$ and $y=0$ an effective power $n_{\mathrm{pai}}=8.3$. Because of the rapidity dependence and the unknown $A$-dependence at $p_{\text {lab }}=200 \mathrm{GeV} / c$ it is not clear if this power is high enough to be in agreement with all the data.

In order to show the dependence of our fit on the structure- and fragmentation functions the various contributions are plotted separately in Fig. 11 for $p_{\text {lab }}=400 \mathrm{GeV} / c$. In the considered $x_{T}$-interval $\left(x_{T} \lesssim 0.3\right)$ the process $q g \rightarrow q g$ is the dominant one, and it has the correct $x_{T}$-dependence. Below $x_{T}=0.2$, the subprocess $g g \rightarrow g g$ is large, larger than the quark-quark scattering terms (including $\bar{q} q \rightarrow \bar{q} q$ and $\bar{q} \bar{q} \rightarrow \bar{q} \bar{q}$ ). Quark-quark scattering becomes important only for $x_{T} \gtrsim 0.25$. The fusion-type processes $g g \rightarrow q \bar{q}$ and $q \bar{q} \rightarrow g g$ give negligible contributions.

The quark-quark term is rather restricted by the neutrino data, and by the electron-position annihilation data as discussed above. The range of $p_{T}$ explored in the considered experiment for $\pi^{+} \pi^{-}$pairs corresponds according to (20) to $20 \lesssim Q^{2} \lesssim 60 \mathrm{GeV}^{2} / \mathrm{c}^{2}$, which is inside the range of $Q^{2}$ covered by the lepton data. However, since the quark-quark term is smaller than the other ones, experimental information on the pair-cross section for $p_{T} \gtrsim 4 \mathrm{GeV} / c$ and $x_{T} \gtrsim 0.25$ would be needed to check more directly the consistency of the QCD-approach. The quark-gluon and the gluongluon term which give large constributions depend crucially on the poorly know $x$-dependence and 


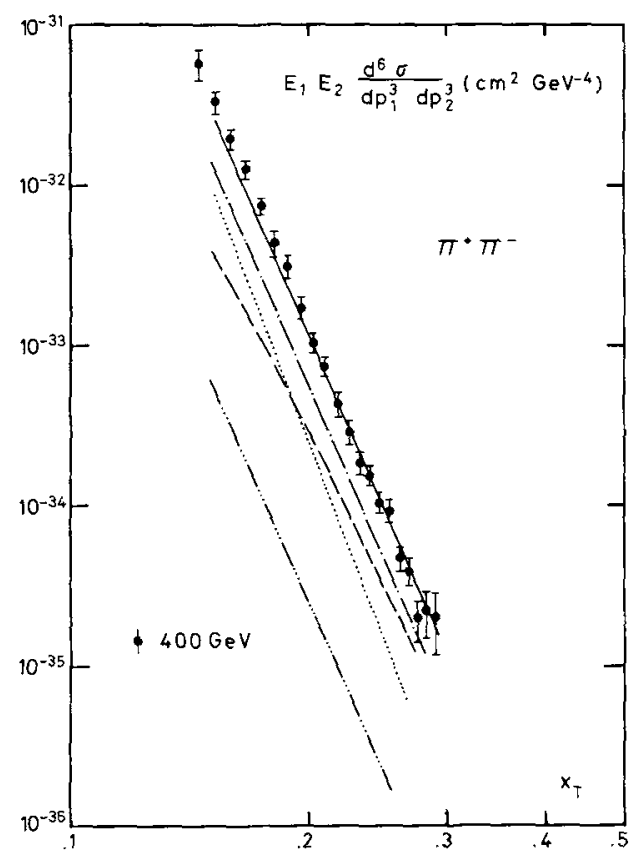

Fig. 11. Contributions of the various QCD subprocesses to the symmetric pair-cross section at $p_{\text {lab }}=400 \mathrm{GeV} / \mathrm{c}$ versus $x_{T}: q g \rightarrow q g(--), g g \rightarrow g g(\ldots), q q \rightarrow q q(--)$ and $g g \rightarrow q \bar{q}(-\ldots-)$. The sum of these contributions is shown by the solid curve. The data at $p_{l z b}=400 \mathrm{GeV} / c$ are taken from [2]

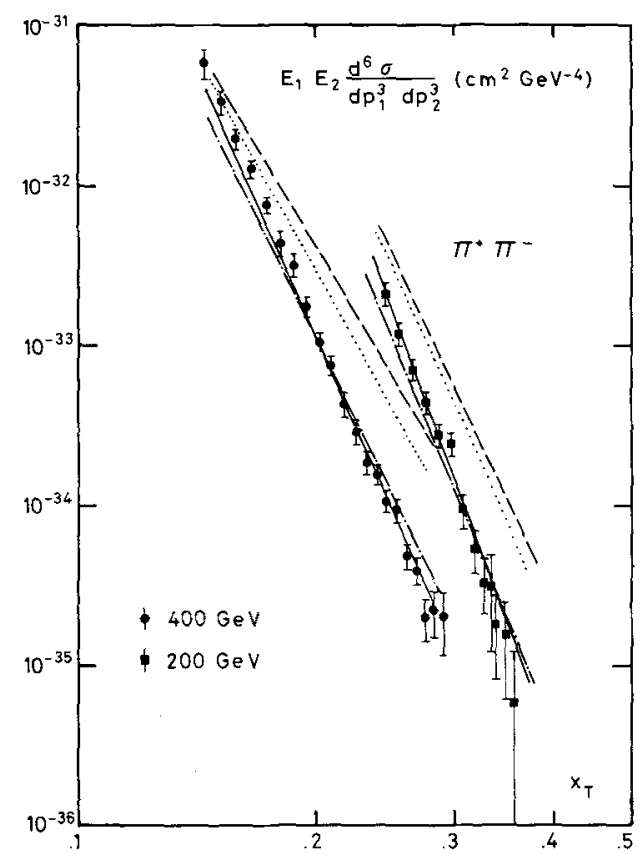

Fig. 12. Illustration of the scale breaking effects in the symmetric pair-cross section at $y=0.0$ and two energies $\left(p_{1 \mathrm{ab}}=200\right.$ and $400 \mathrm{GeV} / c$ ). The scaling formula is given by the dashed curve with $\alpha_{s}=0.4$ and $f\left(x, Q^{2}=5\right)$ and $D\left(z, Q^{2}=25\right)$. The dotted curve results from changing $\alpha_{s}$ into $\alpha_{s}\left(Q^{2}\right)$, the dashed-dotted one from changing in addition $f(x)$ into $f\left(x, Q^{2}\right)$ and the full curve from changing $D(z)$ into $D\left(z, Q^{2}\right)$. The data at $p_{\text {ab }}=200 \mathrm{GeV} / \mathrm{c}$ have $y=0.4$, those at $p_{\mathrm{lab}}=400 \mathrm{GeV} / c$ are at $y=0.0$
$Q^{2}$-dependence of the gluon distribution and fragmentation functions. Using the Buras and Gaemers parametrization [23], which has $M_{3}^{g}\left(Q^{2}=5 \mathrm{GeV}^{2} / c^{2}\right)$ smaller than that deduced from the new neutrino data, the gluon terms are smaller and we would predict a pair-cross section a factor two to three below the $\pi^{+} \pi^{-}$-data. The importance of the quarkgluon term is, however, consistent with the analysis of the large $p_{T}$ single particle spectra [6-10].

In Fig. 12 we examine the scale breaking effects from $\alpha_{s}\left(Q^{2}\right), f\left(x, Q^{2}\right)$ and $D\left(z, Q^{2}\right)$ separately, starting from the scaling formula with $\alpha_{s}=0.4, f\left(x, Q^{2}=5\right)$ and $D\left(z, Q^{2}=25\right)$. In terms of changing the $p_{T}$ dependence parametrized by $p_{T}^{-n_{\text {pair }}}$ at fixed $x_{T}=0.26$ and $y=0$ the following powers are gained: $\Delta n_{\text {pair }}=0.9$ by $\alpha_{s} \rightarrow \alpha_{s}\left(Q^{2}\right), \Delta n_{\text {pair }}=1.2$ by $f(x) \rightarrow f\left(x, Q^{2}\right)$ and $\Delta n_{\text {pair }}=1.2$ by $D(z) \rightarrow D\left(z, Q^{2}\right)$. The main scale breaking is due to the gluons. In Fig. 13 we show the different scale breaking effects for the quark-quark term. We deduce at $x_{T}=0.26$ and $y=0 \Delta n_{\text {pair }}=0.6$ for $f(x) \rightarrow f\left(x, Q^{2}\right)$, and $\Delta n_{\text {pair }}=0.85$ for $D(z) \rightarrow$ $D\left(z, Q^{2}\right)$ leading to a total power of $n_{\text {pair }}=7.35$. Of course, these powers cannot be directly extrapolated to other values of $p_{T}$ and $x_{T}$. In Fig. 14 we show predictions for $p p \rightarrow \pi^{0} \pi^{0} X$ in the symmetric backto-back configuration at $y=0$ for ISR-energies and for a large $p_{T}$ range up to $p_{T}=10 \mathrm{GeV} / c$. Since at these energies the deviations from the $p_{T}^{-8}$ behaviour in the single inclusive $\pi^{0}$-spectra have been

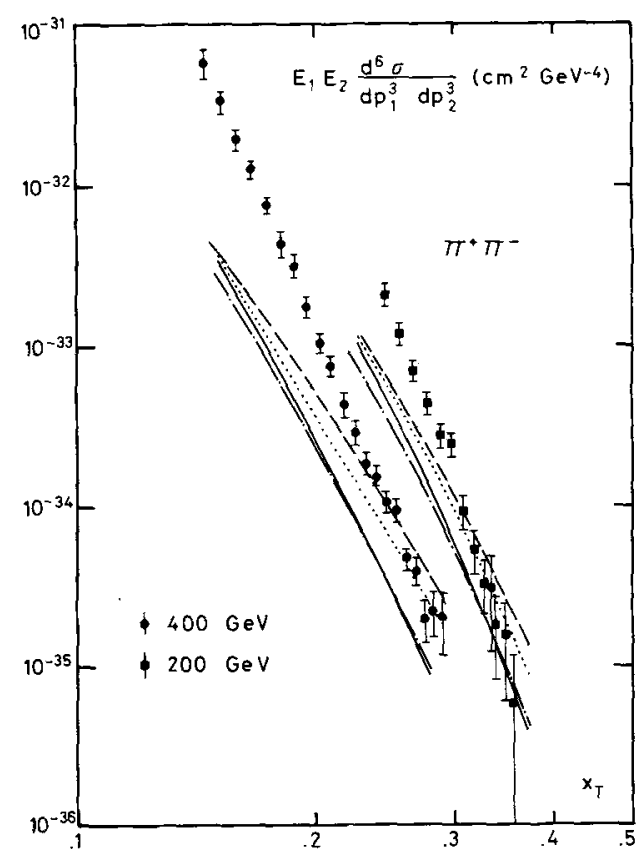

Fig. 13. Illustration of the scale breaking effects in the symmetric pair-cross section, when only the elastic quark-quark subprocess is taken into account. The dashed curve represents the scaling expression with $\alpha_{s}=0.4, f\left(x, Q^{2}=5\right)$ and $D\left(z, Q^{2}=25\right)$. The dotted curve results from changing $\alpha_{s}$ into $\alpha_{s}\left(Q^{2}\right)$, the dashed-dotted one from changing in addition $f(x)$ into $f\left(x, Q^{2}\right)$ and the full curve from changing $D(z)$ into $D\left(z, Q^{2}\right)$. The data are as in Fig. 12 


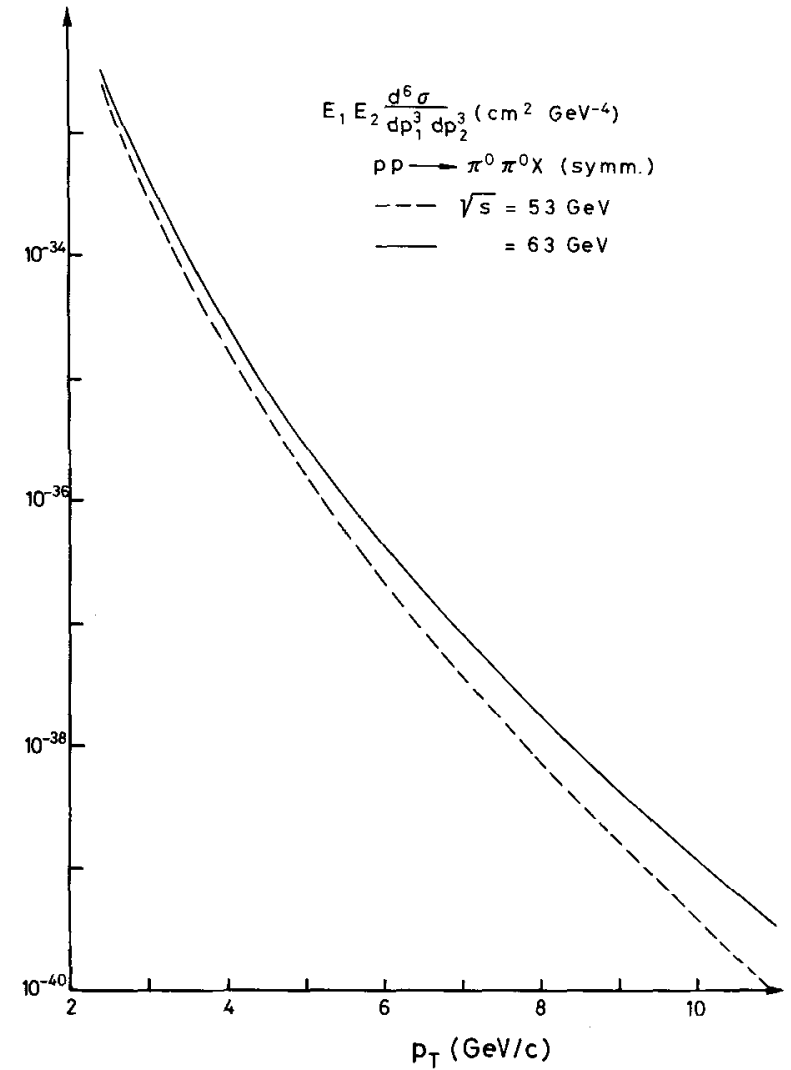

Fig. 14. Prediction of the $Q C D$ model for the symmetric paircross section versus $p_{T}$ for $p p \rightarrow \pi^{0} \pi^{0} X$ at ISR energies, $\sqrt{s}=53$ $\mathrm{GeV}$ (dashed curve) and $\sqrt{s}=63 \mathrm{GeV}$ (solid curve)

measured for the first time $[27,28]$, it seems interesting to look at the $p_{T}$ dependence of the paircorrelations. According to our parametrizations we would expect an effective $p_{T}$-power at $y=0$ for $\sqrt{s}$ of 53 to $63 \mathrm{GeV}$ of $n_{\text {pair }}=7.2$ at $x_{T} \simeq 0.1$ decreasing to $n_{\text {pair }}=6.8$ at $x_{T} \simeq 0.3$.

\section{Quantum Number Correlations}

In order to check further the consistency of the QCD-model with data, we calculate pair-cross sections involving $K^{+}, K^{-}, p$ and $\bar{p}$. For this calculation we need the corresponding fragmentation functions. At $Q^{2}=25 \mathrm{GeV}^{2} / c^{2}$ the following input is used,

$$
\begin{aligned}
& D_{u}^{K^{+}}=D_{\tilde{s}}^{K^{+}}=0.4 D_{u}^{\pi^{+}}, \\
& D_{d}^{K^{+}}=D_{u}^{K^{-}}=D_{d}^{K^{-}}=D_{\bar{s}}^{K^{-}}=0.4 D_{d}^{\pi^{+}}, \\
& D_{g}^{K^{+}}=D_{g}^{K^{-}}=0.4 D_{g}^{\pi^{+}}, \\
& D_{u}^{p}=2 D_{d}^{p}=0.5 \frac{(1+z)}{z}(1-z)^{3}, \\
& D_{u}^{\bar{p}}=2 D_{d}^{\bar{p}}=0.5(1-z)^{4} / z, \\
& D_{g}^{p}=D_{g}^{\bar{p}}=0.29(1-z)^{4} / z .
\end{aligned}
$$

The powers of $(1-z)$ are taken from the counting rules [26]. The normalization parameters of $D_{q, g}^{K^{+}}$are chosen to fit the absolute normalization of the $K^{+} \pi^{-}$ pair data of [3] and the value of around 0.4 is also consistent with the SU(3) breaking observed in other reactions. The normalization of $D_{g}^{p}$ is determined from the momentum sumrule. The $Q^{2}$ dependence is then deduced from the QCD moment equations, in the same way as for the pion fragmentation functions.

The model is expected to have in first approximation the factorization property

$\frac{d \sigma\left(C_{1} D_{1}\right)}{d \sigma\left(C_{2} D_{1}\right)}=\frac{d \sigma\left(C_{1} D_{2}\right)}{d \sigma\left(C_{2} D_{2}\right)}$

However, only the integrands in (10) do factorize for a given subsprocess, and if the fragmentation functions have different shapes, the cross sections do not factorize exactly. We get, e.g.

$$
\frac{d \sigma(p \bar{p})}{d \sigma\left(\pi^{+} \bar{p}\right)} \simeq 1.8 \frac{d \sigma\left(p \pi^{-}\right)}{d \sigma\left(\pi^{+} \pi^{-}\right)} \text {for } 2 \leqq p_{T} \leqq 4 \mathrm{GeV} / c
$$

Furthermore the model predicts a $p_{T}$-dependence at fixed $x_{T}$ which is not very different for different hadron pairs.

A comparison with the data [3] is shown in Fig. 15. It can be seen, that the $\pi^{+} K^{-}$and especially the $p \pi^{-}$spectra have a less steep $p_{T}$-dependence $\left(m^{\prime}=\right.$ $\left|p_{T C}\right|+\left|p_{T D}\right|$ than the data. The $p \pi^{-}$spectra are also lower in normalization than the data. Contributions from CIM subprocesses would be in the right direction to remedy these discrepancies [29]. With respect to the $\Delta p_{T}=\left|p_{T+}\right|-\left|p_{T-}\right|$ dependence one has to remark that the asymmetric behaviour predicted by the model for the $p \pi^{-}$pairs is indicated by the data at $m^{\prime}=6.6 \mathrm{GeV} / c$.

Due to the fact that the data only exist at one energy $\left(p_{\mathrm{lab}}=400 \mathrm{GeV} / c\right)$, the $p_{T}$ and $x_{T}$ dependences cannot be separated. Furthermore nuclear corrections are not made. It would be very interesting to have more extensive data on the quantum number dependence of pair-cross sections in order to check in more detail the above predictions.

\section{Summary and Discussion}

In this paper it is shown that measurements of the cross section for a pair of hadrons produced backto-back at large $p_{T}$, i.e. the symmetric pair-cross section, are extremely useful to test hard scattering models. The inclusion of internal transverse momenta, and the corresponding possible inclusion of parton scattering at low $\hat{t}$ and $\hat{\mathrm{s}}$, which has been much discussed for single particle spectra, are apart from an overall normalization factor unimportant for this cross section already for moderate $p_{T}$. This is shown quantitatively by comparing the results of the simple formula for the pair-cross section in the "hard scattering" limit with Monte Carlo integrations of the full expression containing various assumptions 

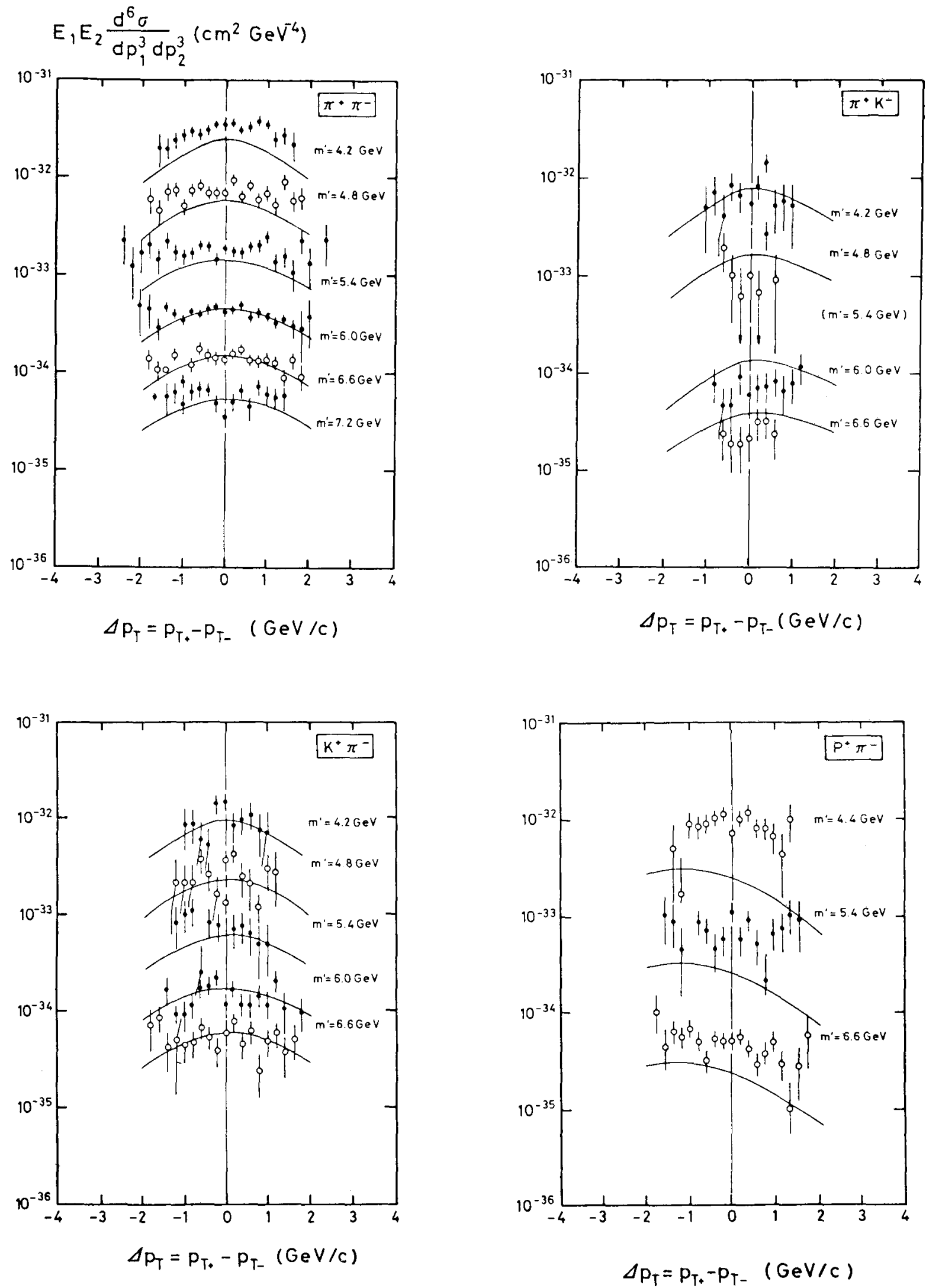

Fig. 15. Comparison of the data [3] for the pair-cross sections for $\pi^{+} \pi^{-}, K^{+} \pi^{-}, \pi^{+} K^{-}$and $p \pi^{-}$pairs as a function of $m^{\prime}=p_{T C}+p_{T D}$ and $\Delta p_{r}=p_{T C}-p_{T D}$ in proton-beryllium collisions at $p_{\text {lab }}=400 \mathrm{GeV} / c$ with the QCD model 
about $\left\langle q_{T}^{2}\right\rangle_{p / h}$ and the cut-off parameter $m_{0}^{2}$ for $\hat{t} \rightarrow \hat{t}-m_{0}^{2}$. The "hard scattering" limit is good within $20-30 \%$ for $p_{\mathrm{T}} \geq 2 \mathrm{GeV} / \mathrm{c}$. For the single particle cross section the results of the hard scattering formula are modified by factors $5-10$ in this range of $p_{T}$, but modifications outside the hard scattering limit are certainly ambiguous. We propose therefore careful measurements of the symmetric pair-cross sections at moderate $p_{T}$ and at several energies, which should test directly the models for the hard cross section of the subprocesses.

One set of data of this kind already exists. As an illustration of the formalism we compare two models, a scaling one (the quark-quark scattering model) and a model based on QCD. For a scaling model the prediction is fairly simple:

$E_{C} E_{D} d^{6} \sigma /\left.d^{3} p_{C} d^{3} p_{D}\right|_{\text {symm }}=\tilde{f}\left(x_{T}\right) / p_{T}^{n_{\text {pair }}}, n_{\text {pair }}=2 n+1$, where $n$ is the inverse power of $\hat{t}$ in the hard scattering cross-section at fixed angle. Because of the unknown $A$-dependence and the possible $y$-dependence of the $p_{\text {lab }}=200 \mathrm{GeV} / c$ data we can only determine $n_{\text {pair }}$ to be between 8 and 10 for $\tilde{f}\left(x_{T}\right)$ given by the model. The normalization of the hard cross-section as determined from the pair data within the phenomenological quark-quark model gives in the "hard scattering" limit for the single particle spectra a contribution below the data by a factor 3-4. The $p_{T}$-dependence is related by $n_{\text {single }}=n_{\text {pair }}-1$. Corrections due to the internal transverse momenta would enhance the magnitude and steepen the single particle cross-section, i.e. $n_{\text {single }}>n_{\text {pair }}-1$.

In the QCD model the predicted single particle spectra in the "hard scattering" limit are far below the data for $p_{T}<6 \mathrm{GeV} / c$. For the pair cross-section we show that the QCD model in the "hard scattering" limit can be made consistent with the data for $\pi^{+} \pi^{-}$ pairs at FNAL energies. A test of our predictions at ISR energies would be needed to confirm this point. A crucial role in our fit is played by the gluon, and the pair cross-section can in fact be a quite sensitive probe of the gluon distribution and fragmentation functions.

A comparison of the QCD model with data on pairs with other quantum numbers shows that the predictions seem in general to be flatter than the data. At the high end of the experimental $p_{T}$-range, $p_{T} \simeq 3.3 \mathrm{GeV} / c$, however, even $p \pi^{-}$pairs are in reasonable agreement with the data. It should be noted that single particle spectra of protons have not been described by QCD-parametrizations [30].

An experiment separating the $x_{T}$ and $p_{T}$-dependence for pairs involving baryons and kaons would be very interesting.

Acknowledgements. It is a pleasure to thank H. Jöstlein and M.L. Good for useful discussions of the data. We thank J. Cleymans for several helpful discussions. One of us (B.P.) did part of this work at MIT, University of Wisconsin and SLAC. He wants to thank these institutes for support and warm hospitality.

\section{References}

1. Berman, S.M., Bjorken, J.D., Kogut, J.B. : Phys. Rev. D4, 3388 (1971);

Ellis, S.D., Kislinger M.B., ibid. D9 2027 (1974);

Sivers, D., Brodsky, S., Blankenbecler, R. : Phys. Rep. 23C, 1 (1976)

2. Jöstlein, H. et al. : Phys. Rev. Lett. 42, 146 (1979)

3. Jöstlein, $\mathbf{H}$. et al. : Inclusive production of large transverse momentum hadrons and hadron pairs, submitted to Phys. Rev.

4. Baier, R., Cleymans, J., Kinoshita, K., Petersson, B. : Nucl. Phys. B118, 139 (1977)

5. Field R.D., Feynman, R.P. : Phys. Rev. D15, 2590 (1977); Feynman, R.P., Field, R.D., Fox, G.C. : Nucl. Phys. B128, 1 (1977)

6. Field, R.D. : Phys. Rev. Lett. 40, 997 (1978);

Feynman, R.P., Field, R.D., Fox, G.C. : Phys. Rev. D18, 3320 (1978);

Field, R.D. : Talk presented at the XIX International Conference on High Energy Physics, Tokyo, 1978, CALT-68-683 (1978)

7. Combridge, B.L., Kripfganz, J., Ranft, J. : Phys. Lett. 70B, 234 (1977)

8. Cutler R., Sivers, D. : Phys. Rev. D17, 196 (1978)

9. Owens, J.F., Reya, E.. Glück, M. : Phys. Rev. D18, 1501 (1978)

10. Contogouris, A.P., Gaskell, R., Papadopoulos, S.: Phys. Rev. D17, 2314 (1978)

11. Bosetti, P.C. et al. : Nucl. Phys. B142, 1 (1978)

12. Groot, J.G.H. de, et al. : Universität Dortmund preprint $12 / 78$; Groot J.G.H. de, et al. : Z. Physik C, Particles and Fields 1 , $143(1979)$

13. Combridge, B.L. : In ISR Discussion Meeting Number 21 (1977)

Kinoshita, K. : In Workshop on large $p_{T}$ phenomena, Bielefeld, BI-TP 77/39 (1977)

14. Halzen, F., Ringland, G.A., Roberts, R.G. : Phys. Rev. Lett. 40, $991(1978)$

Caswell, W.E., Horgan, R.R., Brodsky, S.J. : Phys. Rev. D18, 2415 (1978).

15. Hanson, G. : Proceedings of the XIII Rencontre de Moriond, Vol. II, p. 15 (1977), Tran Thanh Van, ed., Editions Frontieres, Dreux, France

16. PLUTO Collaboration, Berger, Ch. et al. : Phys. Lett. 78B, 1976 (1978)

17. Kaplan D.M. et al. : Phys. Rev. Lett. 40, 435 (1978)

18. Donnachie A. Landshoff, P.V. : Nucl. Phys. B112, 233 (1976)

19. Sehgal, L.M. : Proceedings of the International Symposium on Lepton and Photon Interactions at High Energies, Hamburg 1977, p. 837 (1977)

20. McCarthy, R.L. et al. : Phys. Rev. Lett. 40, 213 (1978)

21. DASP Collaboration, Brandelik, $R$. et al.: Phys. Lett. 67B 363 (1977)

22. Sachrajda, C.T. : Phys. Lett. 76B, 100 (1978)

23. Buras, A.J., Gaemers, K.J.F. : Nucl. Phys. B132, 249 (1976)

24. Politzer, H.D. : Phys. Rep. 14C, 129 (1974)

25. Georgi, H., Politzer, H.D. : Nucl. Phys. B36, 445 (1978); Owens, J.F. : Phys. Lett. 76B, 85 (1978); Uematsu, T. : Phys. Lett. 79B, 97 (1978)

26. Jones, D., Gunion, J.F. : Phys. Rev. D19, 867 (1979)

27. Clark, A.G. et al. : Phys. Lett. 74B, 267 (1978)

28. Angelis A.L.S. et al. : Phys. Lett. 79B, 505 (1978)

29. Gunion, J.F., Petersson, B. : in preparation

30. Owens, J.F.: Florida State University preprint FSU-HEP$781220(1978)$ 